\title{
Review: smoking cessation interventions during pregnancy reduce maternal smoking
}

Lumley J, Oliver S, Waters E. Smoking cessation programs implemented during pregnancy. (Cochrane Review, latest version 25 May 1998). In: Cochrane Library. Oxford: Update Software.

\section{Question}

What is the effectiveness of smoking cessation interventions implemented during pregnancy for reducing maternal smoking during pregnancy and for improving newborn outcomes?

\section{Data sources}

\{Medline was searched using the strategies of the Cochrane Pregnancy and Childbirth Group. Medline, PsycLIT, Datastar, Dissertation Abstracts Online, Applied Social Sciences Index and Abstracts, Social Citations Index, and Social Science Citations Index were searched using the strategies of the Cochrane Tobacco Group. Studies were also identified from handsearches of 60 journals, conference proceedings, bulletins, and bibliographies of review articles $\}^{*}$.

\section{Study selection}

Randomised and quasi-randomised controlled trials of interventions implemented during pregnancy to increase maternal smoking cessation were selected.

\section{Data extraction}

Data were extracted on study design, participants, participation and withdrawal rates, interventions, and outcomes. Methodological quality of studies was assessed.

\section{Main results}

40 trials (1975-97) of healthy pregnant women were identified. Interventions took place in hospitals or community antenatal clinics and included information on the risks of smoking to the fetus and infant and the benefits of stopping; recommendations to stop; feedback about the fetus; and cognitive behavioural strategies for stopping. Main outcome measure was continued maternal smoking in late pregnancy.
Meta-analyses were based on a fixed effects model. Smoking cessation interventions reduced continued maternal smoking during late pregnancy compared with usual care (table). Results were similar when analyses were restricted to 17 trials with biochemically validated smoking cessation, 11 trials of interventions with the highest intensities, and 12 trials with high quality interventions. Mothers who received smoking cessation interventions had fewer low birthweight infants (table) and the infants had a higher mean birth weight ( 8 trials, increase of $41 \mathrm{~g}$, 95\% CI 16.6 to 65.5) compared with mothers who received usual care. There were no group differences in the number of very low birthweight infants ( 3 trials), preterm births ( 5 trials), stillbirths (3 trials), or total perinatal deaths ( 3 trials).

\section{Conclusion}

Smoking cessation interventions implemented during pregnancy reduce maternal smoking during pregnancy and reduce the number of low birthweight infants.

*Information provided by author.

Smoking cessation intervention (SCI) v usual care (UC) during pregnancy

Weighted event rates

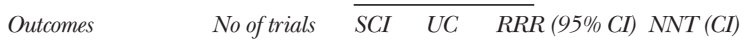

Continued smoking $31(\mathrm{n}=9081) \quad 87 \% \quad 91 \% \quad 7 \%(6$ to 9$) \quad 23$ (18 to 29) Low birth weight $\quad 7(\mathrm{n}=6578) \quad 7.0 \% \quad 8.5 \% \quad 18 \%(3$ to 31$) \quad 68$ (37 to 496$)$

†Abbreviations defined in glossary; RRR, NNT, and CI calculated from data in article.

Sources of funding: Department of Health, UK; Victorian Health Promotion Foundation, Australia.

For correspondence: Prof J Lumley, Centre for the Study of Mothers'and Children's Health, La Trobe University, 463 Cardigan Street, Carlton, Victoria 3053, Australia. Fax +61393481129.

\section{Commentary}

Smoking during pregnancy has been implicated as a cause of low birth weight and intrauterine growth retardation. It is also associated with an increased risk of premature birth, placenta previa, abruptio placenta, and spontaneous abortion. ${ }^{1}$ This systematic review by Lumley et al evaluates the effectiveness of interventions that target smoking cessation during pregnancy. Interventions varied in intensity, duration, and setting. Limitations of studies included in the review were: (a) lack of attention to issues that women have repeatedly stated as reasons for smoking or difficulties with stopping; (b) failure to document smoking relapse rates after delivery; and (c) absence of data on the relation between smoking and infant feeding decisions.

The authors reason that given the effect of maternal smoking on fetal health, evidence of even a small effect is adequate to support the implementation of smoking cessation programmes in all maternity care settings. They also suggest that questions about maternal smoking should become a routine part of antenatal care. Although implementation of these changes is warranted, clinical strategies should reflect our emerging understanding of smoking during pregnancy and postpartum. For example, smoking related questions in antenatal clinic protocols should address smoking not merely as a behaviour but rather as a social phenomenon. Ignoring the influence of partners, families, and close friends in the smoking related decisions of pregnant women weakens the potential effects of interventions. Continuing education for nurses in the effective use of cognitive behavioural strategies is important.

Strategies to reduce postpartum smoking relapse rates, estimated to be as high as $60 \%,{ }^{1}$ should be part of standard prenatal and postnatal care. A recent qualitative study suggests that many women who stop smoking during pregnancy do so for the baby, but not for themselves. ${ }^{2}$ Determining whether women have stopped smoking for themselves or for the baby may help to tailor interventions to the differential needs of pregnant smokers.

Finally, a growing body of literature supports the relation between smoking and infant feeding practices. ${ }^{3}$ Thus, women should be encouraged to discuss how their smoking choices influence their decisions about breast feeding.

Nancy C Edwards, RN, PhD Associate Professor, School of Nursing University of Ottawa Ottawa, Ontario, Canada

1 Edwards N, Sims-Jones N, Hotz S. Pre and postnatal smoking: a review of the literature. Ottawa: Community Health Research Unit Monograph M96-3, 1996.

2 Edwards N, Sims-Jones N. Birth 1998;25:95-

3 Edwards N, Sims-Jones N, Breithaupt K. Can J Nurs Res, 1998;30:83-98. 